\title{
Dengue infections in non-immune travellers to Thailand
}

\author{
E. MASSAD ${ }^{1,2}$, J. ROCKLOV ${ }^{3}$ AND A. WILDER-SMITH ${ }^{3,4 *}$ \\ ${ }^{1}$ School of Medicine, The University of São Paulo and LIM01 HCFMUSP, Brazil \\ ${ }^{2}$ London School of Hygiene and Tropical Medicine, London, UK \\ ${ }^{3}$ Centre for Global Health Research, University of Umea, Sweden \\ ${ }^{4}$ Institute of Public Health, University of Heidelberg, Germany
}

Received 29 December 2011; Final revision 3 March 2012; Accepted 5 March 2012; first published online 3 May 2012

\section{SUMMARY}

Dengue is the most frequent arboviral disease and is expanding geographically. Dengue is also increasingly being reported in travellers, in particular in travellers to Thailand. However, data to quantify the risk of travellers acquiring dengue when travelling to Thailand are lacking. Using mathematical modelling, we set out to estimate the risk of non-immune persons acquiring dengue when travelling to Thailand. The model is deterministic with stochastic parameters and assumes a Poisson distribution for the mosquitoes' biting rate and a Gamma distribution for the probability of acquiring dengue from an infected mosquito. From the force of infection we calculated the risk of dengue acquisition for travellers to Thailand arriving in a typical year (averaged over a 17-year period) in the high season of transmission. A traveller arriving in the high season of transmission and remaining for 7 days has a risk of acquiring dengue of $0 \cdot 2 \%(95 \% \mathrm{CI} 0 \cdot 16-0 \cdot 23)$, whereas the risk for travel of 15 and 30 days' duration is $0.46 \%(95 \%$ CI $0 \cdot 41-0 \cdot 50)$ and $0.81 \%(95 \%$ CI $0 \cdot 76-0.87$ ), respectively. Our data highlight that the risk of non-immune travellers acquiring dengue in Thailand is substantial. The incidence of $0.81 \%$ after a 1 -month stay is similar to that reported in prospective seroconversion studies in Israeli travellers to Thailand, highlighting that our models are consistent with actual data. Risk estimates based on mathematical modelling offer more detailed information depending on various travel scenarios, and will help the travel medicine provider give better evidence-based advice for travellers to dengue-endemic countries.

Key words: Dengue, mathematical modelling, risk estimates, Thailand, travellers.

\section{INTRODUCTION}

Dengue is a flaviviral infection transmitted by Aedes mosquitoes. The dengue viruses are among the most widespread geographically of the arboviruses and are found in tropical and subtropical areas where $2 \cdot 5-3$

\footnotetext{
* Author for correspondence: Professor A. Wilder-Smith, Institute of Public Health, Im Neuenheimer Feld 365, University of Heidelberg, 69120 Heidelberg, Germany. (Email: epvws@pacific.net.sg)
}

billion people are at risk of infection [1]. The past two decades have seen an unprecedented geographical expansion of dengue, the reasons of which are complex and include increasing urbanization, lack of effective vector control, climate change and international travel [2].

Many of the dengue endemic countries are popular tourist destinations and dengue has emerged as a frequent problem in international travellers [3]. GeoSentinel is a global network of travel medicine

The online version of this article is published within an Open Access environment subject to the conditions of the Creative Commons Attribution-NonCommercial-ShareAlike licence $<$ http://creativecommons.org/licenses/by-nc-sa/2.5/ $>$. The written permission of Cambridge University Press must be obtained for commercial re-use. 
providers [4] and dengue accounts for up to $2 \%$ of all illness in returned travellers who visit GeoSentinel clinics [5]. The GeoSentinel surveillance network has shown that over the past decade dengue has become a more frequent diagnosis than malaria in ill returned travellers from all tropical regions outside of Africa [6]. Risk factors for acquiring dengue depend on duration of travel, season and destination $[5,7]$.

Most of dengue virus infections in travellers are acquired in Asia, followed by the Americas and only a small proportion in Africa [6-8]. Within Asia, Thailand is one of the countries with the highest risk of travellers acquiring dengue [5]. However, these data are based on a surveillance system that depend on returning travellers who seek healthcare (during or post-travel) at GeoSentinel sites and therefore the true incidence of dengue in travellers cannot be determined due to the lack of a denominator.

Risk estimates can be calculated for dengueendemic countries provided that local data on the force of infection and variations over time are available. We used mathematical models to estimate the risk of non-immune persons acquiring dengue when travelling to Thailand.

\section{METHODS}

We obtained data on national epidemiology of dengue in Thailand from 1990 until 2007 which are freely available on the website of the South East Asia Regional Office of the World Health Organization (SEARO) (http://www.searo.who.int/LinkFiles/Dengue_ dengue_Thailand.pdf).

We calculated the average risk for a non-immune traveller who arrives in Thailand in four different moments of time, i.e. in the dry season (winter months), spring (spring months), the wet season (summer) and autumn. We used an adaptation of the model developed by Massad et al. [9] to describe the dynamics of dengue. The model estimates the force of infection, which means the incidence density rate (per capita number of new cases per year) for the period [10]. The force of infection is defined as the per capita number of new cases per time unit as

$h(t)=a b \frac{I_{\mathrm{M}}(t)}{N_{\mathrm{H}}(t)}$,

where $N_{\mathrm{H}}$ is the total human population, $I_{\mathrm{M}}(t)$ is the number of infected mosquitoes, $a$ is the mosquito biting rate and $b$ is the probability that an infectious mosquito will infect a human susceptible.
We calculated the probability of an individual acquiring dengue, $\pi_{\text {travellers }}$, after arriving in Thailand, depending upon the length of stay (for $\omega$ days) and also depending on the time of arrival (at day $\Omega$ ), using the following equation:

$\pi_{\text {travellers }}=\frac{\int_{\Omega}^{\Omega+\omega} S_{\mathrm{H}}(t) h(t) \mathrm{d} t}{N_{\mathrm{H}}(\Omega)}$.

In the interval between $\Omega$ and $\Omega+\omega$, a total of $\int_{\Omega}^{\Omega+\omega}$ $S_{\mathrm{H}}(t) h(t) \mathrm{d} t$ new infections occur. This quantity divided by the total number of individuals in the population at time $\Omega$ gives the probability $\pi_{\text {travellers }}$ above. The model is deterministic with stochastic parameters and assumes a Poisson distribution for the mosquito biting rate, $a$, and a Gamma distribution for the probability of acquiring dengue from an infected mosquito, $b$. We used stochastic methods since the relatively small number of travellers is associated with random fluctuations of the biting rate and therefore fluctuations in the probability of infection should be considered. The models and the variables for the models are described in the Appendix. The parameters applied for the numerical simulations are shown in Table 1.

From the force of infection we calculated the risk of acquiring dengue for travellers to Thailand arriving in a typical year (averaged over a 17-year period).

\section{RESULTS}

Figure 1 shows how the model fits into the actual data (averaged over a 17-years period) of the national epidemiology of Thailand. In Figure 2 we show the model's simulation for non-infected and infected mosquitoes along with the definition of 'seasons': winter is the dry season, summer is the rainy reason, spring and autumn are the interim seasons approximately corresponding with the calendar months of the Northern hemisphere.

Our modelling showed that a traveller arriving in the season of highest transmission (designated 'autumn' and representing the period of the year between the wet and the dry season) who has a stay of 1 week has a risk of acquiring dengue of $0.2 \%(95 \% \mathrm{CI}$ $0 \cdot 16-0 \cdot 23)$, whereas the risk for travel of 2 and 4 weeks' duration is $0.43 \%(95 \%$ CI $0.41-0.50)$ and $0.81 \%(95 \%$ CI $0 \cdot 76-0 \cdot 87)$, respectively (Table 2$)$. Table 1 also shows the risk for the rainy and dry season for 1, 2, 3 and 4 weeks of travel duration. 
Table 1. Model parameters, biological meaning, values and sources

\begin{tabular}{llll}
\hline \hline Parameter & Meaning & Value & Source \\
\hline$a$ & Average daily biting rate & $0 \cdot 164$ & {$[15]$} \\
$b$ & Fraction of actually infective bites & $0 \cdot 088$ & Fitted to data \\
$\mu_{\mathrm{H}}$ & Human natural mortality rate & $3 \cdot 5 \times 10^{-5} \mathrm{day}^{-1}$ & {$[16]$} \\
$r_{\mathrm{H}}$ & Human birth rate & $8 \mathrm{day}^{-1}$ & {$[16]$} \\
$\kappa_{\mathrm{H}}$ & Human carrying capacity & $16 \times 10^{6}$ & {$[16]$} \\
$\alpha_{\mathrm{H}}$ & Dengue mortality in humans & $10^{-3} \mathrm{day}^{-1}$ & {$[17]$} \\
$\gamma_{\mathrm{H}}$ & Human recovery rate & $0 \cdot 143 \mathrm{day}^{-1}$ & {$[17]$} \\
$p_{\mathrm{S}}$ & Susceptible eggs hatching rate & $0 \cdot 15 \mathrm{day}^{-1}$ & {$[18]$} \\
$d 1$ & Winter modulation parameter & $0 \cdot 07$ & Assumed \\
$d_{2}$ & Winter modulation parameter & $0 \cdot 06$ & Assumed \\
$\gamma_{\mathrm{M}}$ & Mosquito latency rate & $0 \cdot 143 \mathrm{day}^{-1}$ & - \\
$f$ & Frequency of seasonal cycles & $2 \cdot 8 \times 10^{-3} \mathrm{day}^{-1}$ & Assumed \\
$\mu_{\mathrm{M}}$ & Mosquito natural mortality rate & $0 \cdot 263 \mathrm{day}^{-1}$ & {$[19]$} \\
$\alpha_{\mathrm{M}}$ & Dengue mortality in mosquitoes & Negligible & - \\
$r_{\mathrm{M}}$ & Oviposition rate & $50 \mathrm{day}^{-1}$ & {$[19]$} \\
$p_{\mathrm{I}}$ & Infected eggs hatching rate & $0 \cdot 15 \mathrm{day}^{-1}$ & {$[19]$} \\
$g$ & Proportion of infected eggs & $0 \cdot 5$ & Assumed \\
$\kappa_{\mathrm{E}}$ & Egg carrying capacity & $9 \cdot 8 \times 10^{7}$ & Assumed \\
$\mu_{\mathrm{E}}$ & Egg natural mortality rate & $0 \cdot 1 \mathrm{day}^{-1}$ & {$[19]$} \\
$c$ & Aedes aegypti susceptibility to dengue & $0 \cdot 087$ & Fitted to data \\
\hline \hline
\end{tabular}

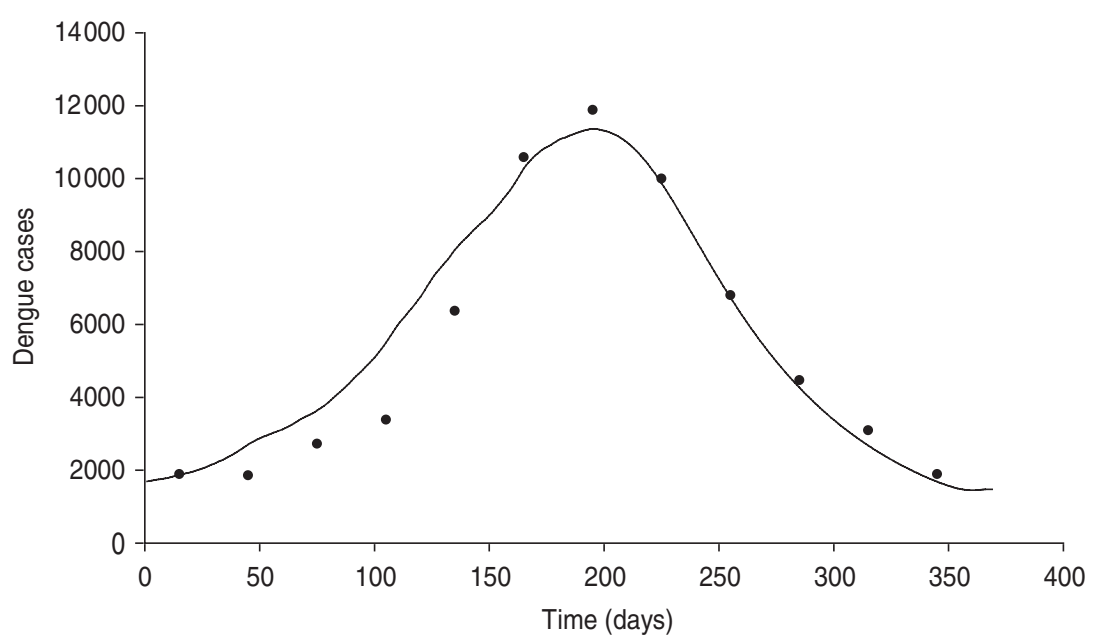

Fig. 1. The model's fit to the actual national epidemiology of dengue in Thailand averaged over 17 years, from 1990 to 2007. Symbols ( ) represent actual data [data from the national epidemiology of Thailand as obtained from the South East Asia Regional Office of the World Health Organization (http://www.searo.who.int/LinkFiles/Dengue_dengue_Thailand.pdf)]. The continuous line (-) represents data obtained from our mathematical model.

Figure 3 presents the result of risk estimation for travellers arriving in each of the four seasons in a logarithmic scale depending on length of stay in Thailand.

\section{DISCUSSION}

Previous studies have attempted to determine the cumulative risk of acquiring dengue in travellers
[7, 11], but such studies do not take into account the seasonality and year to year oscillation of dengue, nor the duration of travel. Most reports are limited to passive surveillance or sentinel surveillance and may therefore underestimate the true risk. First of all, dengue is a disease of short duration and travellers may therefore not seek healthcare in their countries of origin. Second, dengue is not a legally notifiable disease in most countries where travellers originate from. 
Table 2. Risk of acquiring dengue in travellers to Thailand depending on season and duration of travel average risk of dengue in percentage

\begin{tabular}{lllll}
\hline \hline Season/time in the area & 1 week & 2 weeks & 3 weeks & 4 weeks \\
\hline Winter (dry season) & $0 \cdot 000082$ & $0 \cdot 000146$ & $0 \cdot 000202$ & $0 \cdot 000353$ \\
Spring (interim period) & $0 \cdot 00756$ & $0 \cdot 0296$ & $0 \cdot 0555$ & $0 \cdot 0965$ \\
Summer (wet season) & $0 \cdot 0492$ & $0 \cdot 111$ & $0 \cdot 197$ & $0 \cdot 29$ \\
Autumn (following wet season) & $0 \cdot 199$ & $0 \cdot 425$ & $0 \cdot 599$ & $0 \cdot 81$ \\
\hline \hline
\end{tabular}

All values given are percentages.

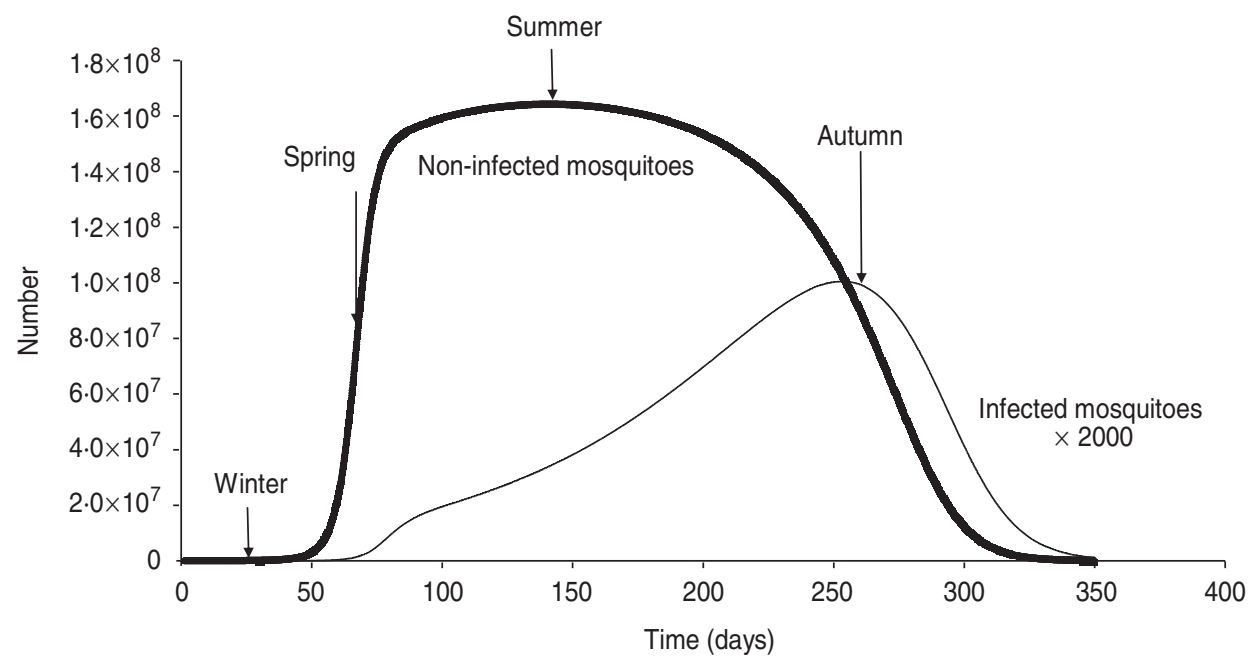

Fig. 2. The model's simulation for non-infected (thick line) and infected (thin line) mosquitoes along with the definition of 'seasons': winter is the dry season, summer is the rainy reason, spring and autumn are the interim seasons approximately corresponding with the calendar months of the Northern hemisphere.

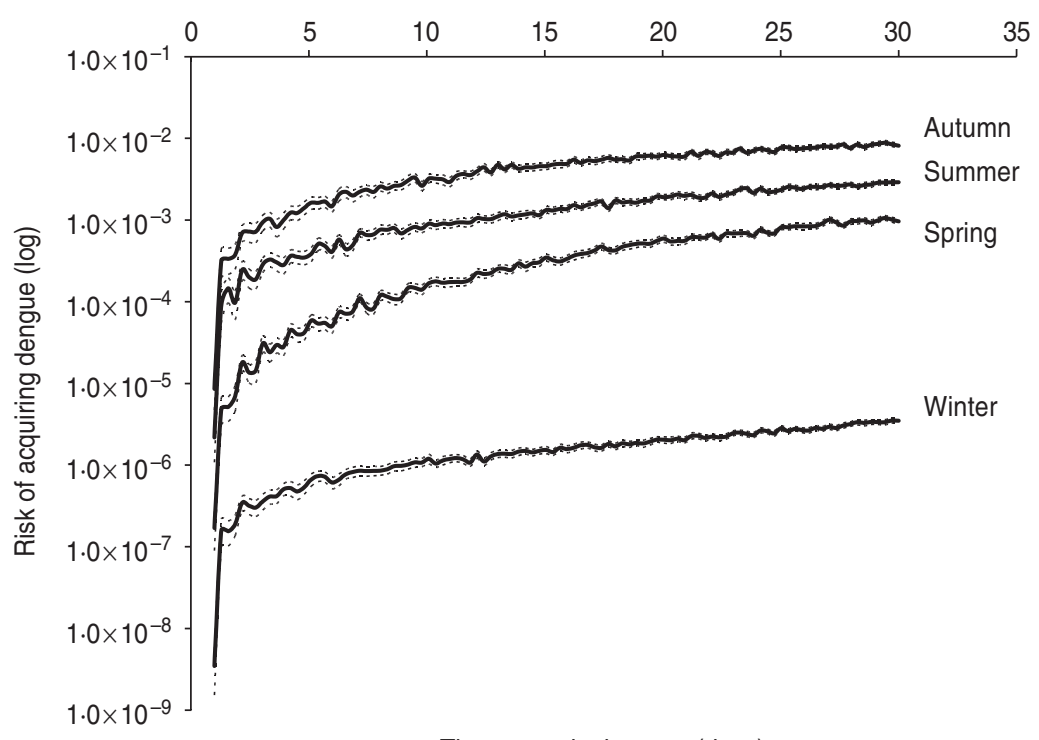

Time spent in the area (days)

Fig. 3. Continuous lines represents the average of 1000 stochastic simulations of the model. Dotted lines represent the $95 \%$ confidence intervals. 
Last, passive surveillance only takes into account symptomatic disease. However, asymptomatic dengue infections are also important in travellers as repeat travel may expose a previously infected traveller to more severe disease [3].

Our data highlight that the risk of non-immune travellers acquiring dengue in Thailand is substantial and varies with season and duration of travel. The difference in incidence between the low and peak season is more than 100 -fold. The increase in incidence is proportional with the length of stay. During the high season for a 4-week stay in Thailand, we estimated a risk of $0.81 \%$ in non-immune travellers. Such an incidence is substantial. During the dry season, the typical travel season, the incidence is $0.0003 \%$. The incidence of $0.81 \%$ after 1 month's travel during the high-risk reason is very similar to that found in two prospective seroconversion studies in Israeli and Dutch travellers [7, 11]. During a mean of 6 months' stay in Israeli travellers in Thailand the seroconversion rate was $6.7 \%$ - and these data were from 1998, a high epidemic year [11]. Probable dengue infection was found in 13/447 Dutch travellers to all dengue-endemic countries (incidence rate 30/1000 person-months) [7]. The fact that our study results based on mathematical models is in keeping with prospective seroconversion studies underlines that our model reflects actual data and can therefore also be applied to other dengue-endemic countries. However, mathematical models in contrast to seroconversion studies, offer additional information taking into account various travel scenarios such as seasonality and duration of travel.

The Dutch study showed an asymptomatic/symptomatic ratio of $3 \cdot 3 / 1$ [7]. Our study considered all dengue infections (symptomatic and asymptomatic) in a non-immune population. The incidence of symptomatic dengue is therefore about three times lower than the actual numbers calculated in our models, but it should be taken into account that the ratio of asymptomatic to symptomatic dengue may vary between 1 and 10 dependent on age, host factors and geography [12].

Using similar mathematical models we found that the risk of acquiring dengue for travellers to Thailand is far higher compared to travellers to Singapore [13]. This finding is consistent with the fact that dengue is more frequent in Thailand than in Singapore [14].

Quantifying the risk of dengue in travellers is an important piece of information to the travel medicine provider and traveller alike. Risk estimates based on mathematical modelling will help the travel medicine provider give better evidence-based advice to travellers to dengue-endemic countries.

\section{APPENDIX}

\section{Equations for the model}

$$
\left.\begin{array}{rl}
\frac{\mathrm{d} S_{\mathrm{H}}}{\mathrm{d} t}= & -a b I_{\mathrm{M}} \frac{S_{\mathrm{H}}}{N_{\mathrm{H}}}-\mu_{\mathrm{H}} S_{\mathrm{H}}+\sigma_{\mathrm{H}} R_{\mathrm{H}}+r_{\mathrm{H}} N_{\mathrm{H}}\left(1-\frac{N_{\mathrm{H}}}{\kappa_{\mathrm{H}}}\right) \\
\frac{\mathrm{d} I_{\mathrm{H}}}{\mathrm{d} t}= & a b I_{\mathrm{M}} \frac{S_{\mathrm{H}}}{N_{\mathrm{H}}}-\left(\mu_{\mathrm{H}}+\gamma_{\mathrm{H}}+\alpha_{\mathrm{H}}\right) I_{\mathrm{H}} \\
\frac{\mathrm{d} R_{\mathrm{H}}}{\mathrm{d} t}= & \gamma_{\mathrm{H}} I_{\mathrm{H}}-\mu_{\mathrm{H}} R_{\mathrm{H}}-\sigma_{\mathrm{H}} R_{\mathrm{H}} \\
\frac{\mathrm{d} S_{\mathrm{M}}}{\mathrm{d} t}= & -\mu_{\mathrm{M}} S_{\mathrm{M}}-a c S_{\mathrm{M}} \frac{\left(I_{\mathrm{H}}+I_{\mathrm{H}}^{\prime}\right)}{N_{\mathrm{H}}} \\
& +r_{\mathrm{M}} N_{\mathrm{M}}\left(1-\frac{N_{\mathrm{M}}}{\kappa_{\mathrm{M}}}\right)\left[c_{s}-\mathrm{d}_{\mathrm{s}} \sin (2 \pi f t)\right] \\
\frac{\mathrm{d} L_{\mathrm{M}}}{\mathrm{d} t}= & a c S_{\mathrm{M}} \frac{\left(I_{\mathrm{H}}+I_{\mathrm{H}}^{\prime}\right)}{N_{\mathrm{H}}} \\
& -\mathrm{e}^{-\mu_{\mathrm{M}} \tau} a c S_{\mathrm{M}}(t-\tau) \frac{\left[I_{\mathrm{H}}(t-\tau)+I_{\mathrm{H}}^{\prime}(t-\tau)\right]}{N_{\mathrm{H}}(t-\tau)}-\mu_{\mathrm{M}} L_{\mathrm{M}} \\
\frac{\mathrm{d} I_{\mathrm{M}}}{\mathrm{d} t}= & \mathrm{e}^{-\mu_{\mathrm{M}} \tau} a c S_{\mathrm{M}}(t-\tau) \frac{\left[I_{\mathrm{H}}(t-\tau)+I_{\mathrm{H}}^{\prime}(t-\tau)\right]}{N_{\mathrm{H}}(t-\tau)}-\mu_{\mathrm{M}} I_{\mathrm{M}}
\end{array}\right\}
$$

In addition, we separated a cohort (denoted by primes and called 'probe') that is followed through the entire outbreak and that is used to calculate the probability that an individual gets dengue infection, according to equation (2), described in the main text.

The evolution equations for the probe cohort are:

$$
\left.\begin{array}{rl}
\frac{\mathrm{d} S_{\mathrm{H}}^{\prime}}{\mathrm{d} t} & =\left(-a^{\prime} b^{\prime} I_{\mathrm{M}} \frac{S_{\mathrm{H}}^{\prime}}{N_{\mathrm{H}}}-\mu_{\mathrm{H}} S_{\mathrm{H}}^{\prime}\right) \theta\left(t-t_{0}\right) \\
\frac{\mathrm{d} I_{\mathrm{H}}^{\prime}}{\mathrm{d} t} & =\left(a^{\prime} b^{\prime} I_{\mathrm{M}} \frac{S_{\mathrm{H}}^{\prime}}{N_{\mathrm{H}}}-\left(\mu_{\mathrm{H}}+\gamma_{\mathrm{H}}+\alpha_{\mathrm{H}}\right) I_{\mathrm{H}}^{\prime}\right) \theta\left(t-t_{0}\right) \\
\frac{\mathrm{d} R_{\mathrm{H}}^{\prime}}{\mathrm{d} t} & =\left(\gamma_{\mathrm{H}} I_{\mathrm{H}}^{\prime}-\mu_{\mathrm{H}} R_{\mathrm{H}}^{\prime}-\sigma_{\mathrm{H}} R_{\mathrm{H}}^{\prime}\right) \theta\left(t-t_{0}\right)
\end{array}\right\}
$$

for

$a^{\prime}=\operatorname{Poisson}(0 \cdot 164)$

$b^{\prime}=\operatorname{Gamma}(0 \cdot 088,0 \cdot 0003)$,

$\left.\begin{array}{l}N_{\mathrm{H}}=S_{\mathrm{H}}+I_{\mathrm{H}}+R_{\mathrm{H}} \\ N_{\mathrm{H}}=N_{\mathrm{H}}+S_{\mathrm{H}}+I_{\mathrm{H}}+R_{\mathrm{H}} \\ N_{\mathrm{M}}=S_{\mathrm{M}}+L_{\mathrm{M}}+I_{\mathrm{M}}\end{array}\right\}$

and $\theta\left(t-t_{0}\right)$ is the Heaviside function.

The parameters' biological significance and the values used in the numerical simulation of the model are shown in Table 1.

We introduced the term $\left[c_{s}-d_{s} \sin (2 \pi f t)\right]$ in the susceptible mosquito population in order to simulate seasonality in the mosquito population. The 
parameters $c_{s}$ and $d_{s}\left(c_{s}>d_{s}\right)$ modulate the intensity of the seasonality, mimicking deep or light winters, depending on the difference between those parameters' values.

\section{ACKNOWLEDGEMENTS}

This research was undertaken by partners from the 'DengueTools' consortium [15]. DengueTools is funded under the Health theme of the Seventh Framework Programme of the European Community, Grant Agreement Number: 282589.

\section{DECLARATION OF INTEREST}

None.

\section{REFERENCES}

1. Gubler DJ. The global emergence/resurgence of arboviral diseases as public health problems. Archives of Medical Research 2002; 33: 330-342.

2. Wilder-Smith A, Gubler DJ. Geographic expansion of dengue: the impact of international travel. Medical Clinics of North America 2008; 92: 1377-1390.

3. Wilder-Smith A, Schwartz E. Dengue in travellers. New England Journal of Medicine 2005; 353: 924-932.

4. Freedman DO, et al. GeoSentinel: the global emerging infections sentinel network of the International Society of Travel Medicine. Journal of Travel Medicine 1999; 6: 94-98.

5. Schwartz E, et al. Seasonality, annual trends, and characteristics of dengue among ill returned travellers, 1997-2006. Emerging Infectious Diseases 2008; 14: 1081-1088.

6. Freedman DO, et al. Spectrum of disease and relation to place of exposure among ill returned tra- vellers. New England Journal of Medicine 2006; 354 : 119-130.

7. Cobelens FG, et al. Incidence and risk factors of probable dengue virus infection among Dutch travellers to Asia. Tropical Medicine \& International Health 2002; 7 : 33133-8.

8. Jelinek T, et al. Epidemiology and clinical features of imported dengue fever in Europe: sentinel surveillance data from TropNetEurop. Clinical Infectious Diseases 2002; 35: 1047-1052.

9. Massad E, et al. The risk of chikungunya fever in a dengue-endemic area. Journal of Travel Medicine 2008; 15: 147-155.

10. Burattini MN, et al. Modelling the control strategies against dengue in Singapore. Epidemiology and Infection 2008; 136: 3109-3119.

11. Potasman I, Srugo I, Schwartz E. Dengue seroconversion among Israeli travellers to tropical countries. Emerging Infectious Diseases 1999; 5: 824-827.

12. Guzman MG, Kouri G. Dengue: an update. Lancet Infectious Diseases 2002; 2: 33-42.

13. Massad E, Wilder-Smith A. Risk estimates of dengue in travellers to dengue endemic areas using mathematical models. Journal of Travel Medicine 2009; 16: 191-193.

14. Wilder-Smith A, et al. Serological evidence for the cocirculation of multiple dengue virus serotypes in Singapore. Epidemiology and Infection 2005; 133: 667-671.

15. Wilder-Smith A, et al. DengueTools: strategies and tools for the surveillance and control of dengue. Global Health Action (in press).

16. Index Mundi. (http://www.indexmundi.com $/ \mathrm{map} /$ ? $\mathrm{v}=$ $30 \& 1=p t$ ). Accessed 18 August 2011.

17. Halstead SB. Dengue. In: Warren KS, Mahmoud AAF, eds. Tropical and Geographical Medicine. New York: McGraw-Hill, 1990, pp. 675-684.

18. Forattini OP. Medical Culicidology. São Paulo: EDUSP, 1996.

19. Brownstein JS, Heth E, O'Neill L. The potential of virulent Wolbachia to modulate disease transmission by insects. Journal of Invertebrate Pathology 2003; 84: 24-29. 